Ann. Biol. anim. Bioch. Biophys., 1979, 19 (3 A), 619-624.

\title{
Défaunation du rumen de mouton
}

\author{
par J. P. JOUANY, J. SENAUD * \\ arec la collaboration fechnique de J. LEFAIVRE et A. VIZET \\ Laboratoire de la Digestion des Ruminants, I.N.R.A., \\ Theix, Saint-Genès-Champanelle, 63110 Beaumont \\ * Laboratoire associé au CNRS no 138, Université de Clermont II. \\ Les Cézeaux B.P. 45, 63170 Aubière.
}

Summary. Defaunation of the sheep rumen.

This reports describes a method for defaunating the sheep rumen. The rumen was emptied by means of a fistula and washed with lukewarm water. A diluted solution of formaldehyde was then introduced and the rumen wall quickly washed. The animals thus treated were fasted for $\mathbf{8 4}$ hrs following the first emptying. They were ready for use in digestive studies 3 weeks after food intake was resumed. This method was reliable and did not disturb the animals as much as some other methods tested (Becker, Schulz and Emmerson, 1929, 1930 ; Abou Akkada et al., 1968). The method of Williams and Dinusson (1972) is accurate but difficult to use because it is only suitable for young lambs.

L'étude du rôle des protozoaires ciliés du rumen dans la digestion des aliments passe par l'utilisation d'animaux à faune contrôlée. Ces derniers sont obtenus en implantant une ou plusieurs espèces de ciliés qui ont été isolées sous une loupe binoculaire, dans le rumen d'animaux préalablement débarrassé de la totalité des protozoaires (défauné).

Plusieurs techniques de défaunation sont à ce jour connues. Les plus nombreuses (Becker, Schulz et Emmerson, 1929-1930 ; Eadie et Oxford, 1954 ; Abou Akkada ef al., 1968 ; Clarke et Reid, 1969 ; Wright et Curtis, 1976) sont fondées sur l'introduction d'agents chimiques (sulfate de cuivre ; benzopyrrole ou méthyl indole ; dioctylsulfosuccinate de sodium ; 1-2 diméthyl-5 nitroimidazole) à des doses toxiques pour la faune du rumen. D'autres auteurs ont pu supprimer les ciliés du rumen, ou tout au moins en réduire le nombre de manière appréciable, en distribuant au Ruminant des rations très digestibles, dont le temps de séjour dans le rumen est très court (Eadie, Hobson etMann, 1967 ; Eadie et al., 1970).

Enfin, la méthode qui est aujourd'hui la plus utilisée et la plus sûre consiste à isoler les jeunes animaux quelques heures après leur naissance. Ils sont alors élevés dans les locaux où ne séjournent pas de Ruminants adultes (Kurihara et al., 1968 ; Eadie et Gill, 1971 ; Senaud et al., 1973 ;Williams et Dinusson, 1973). 
Nous proposons une technique qui permet d'obtenir rapidement et d'une manière sûre, des moutons dont le rumen est totalement dépourvu de protozoaires ciliés, sans pour aułant altérer profondément l'état sanitaire des animaux : la reprise de l'ingestion des aliments s'effectue spontanément, en même temps que la motricité du rumen redevient normale. Les animaux présentent rapidement un état général satisfaisant.

\section{Technique de défaunation (fig. 1).}

Les moutons porteurs de fistules permanentes du rumen, sont mis à jeun pendant $16 \mathrm{~h}$. Nous enlevons alors la canule du rumen, puis nous vidons l'organe de la totalité de son contenu. Nous lavons l'intérieur du rumen avec de l'eau maintenue à $39^{\circ} \mathrm{C}$ et sous pression, à l'aide d'un tuyau branché sur un robinet mélangeur, pendant environ $5 \mathrm{mn}$, jusqu'à ce que l'effluent soit parfaitement clair.

Une solution de formol (50 ml de formol à 30 p. 100 dans 10 l d'eau) est ensuite introduite dans le rumen de façon à mouiller toute la surface de la muqueuse, puis elle est rapidement évacuée en soulevant l'abdomen des animaux en position debout. L'intérieur de l'organe est alors abondamment rincé avec de l'eau tiède pendant $5 \mathrm{mn}$ pour éliminer tout le formol introduit. La canule est ensuite remise en place lorsque le rumen contient environ 51 d'eau claire. Dès cet instant, les animaux sont maintenus à jeun ; seule l'eau reste à leur disposition. On répète ces opérations pendant les 2 jours suivants. La première distribution d'aliment n'intervient que $36 \mathrm{~h}$ après le dernier lavage.

Une fois par jour, pendant toute la période de jeûne, il est nécessaire d'injecter par voie intramusculaire une association de vitamine $B$ (Terneurine ND( $\left.{ }^{1}\right)$ ) en alternance avec un complexe vitaminique $B\left({ }^{2}\right)$ ainsi qu'une ampoule d'extrait autolytique de poissons (Dynalyse forte ND (3)). Nous introduisons également dans le rumen, 2 fois par jour, $200 \mathrm{ml}$ d'une solution d'acide acétique à 5 p. $100(\mathrm{~V} / \mathrm{V})$ ainsi qu'un demi-sachef de transitonyl (ND) $\left(^{3}\right)$ (propionate de sodium).

Les animaux acquièrent en $24 \mathrm{~h}$ une flore abondante qui, dans les jours suivants, atteint une concentration identique à celle observée chez des animaux conventionnels (Zainab, 1978). L'implantation d'une telle flore permet la reprise des processus digestifs et, par là même, la reprise de l'ingestion d'aliments (Hungate, 1966). La contamination bactérienne des animaux défaunés s'effectue à la fois par l'air ambiant ef par

(1) Laboratoire Allard, 10, av. de Messine, 75008 Paris.

(2) Laboratoire Sofca, 28, bd du Parc, 92521 Neuilly.

(3) Lathevet, 20, rue des Fossés St-Jacques, 75005 Paris.

FIG. 1. - Description de la technique de défaunation du rumen de mouton : a : vidage du rumen ; $b$ : sortie de la muqueuse ; $c$ : addition d'une solution diluée de formol et lavage du rumen ; $d$ : prélèvement du contenu de rumen pour contrôler la défaunation. 

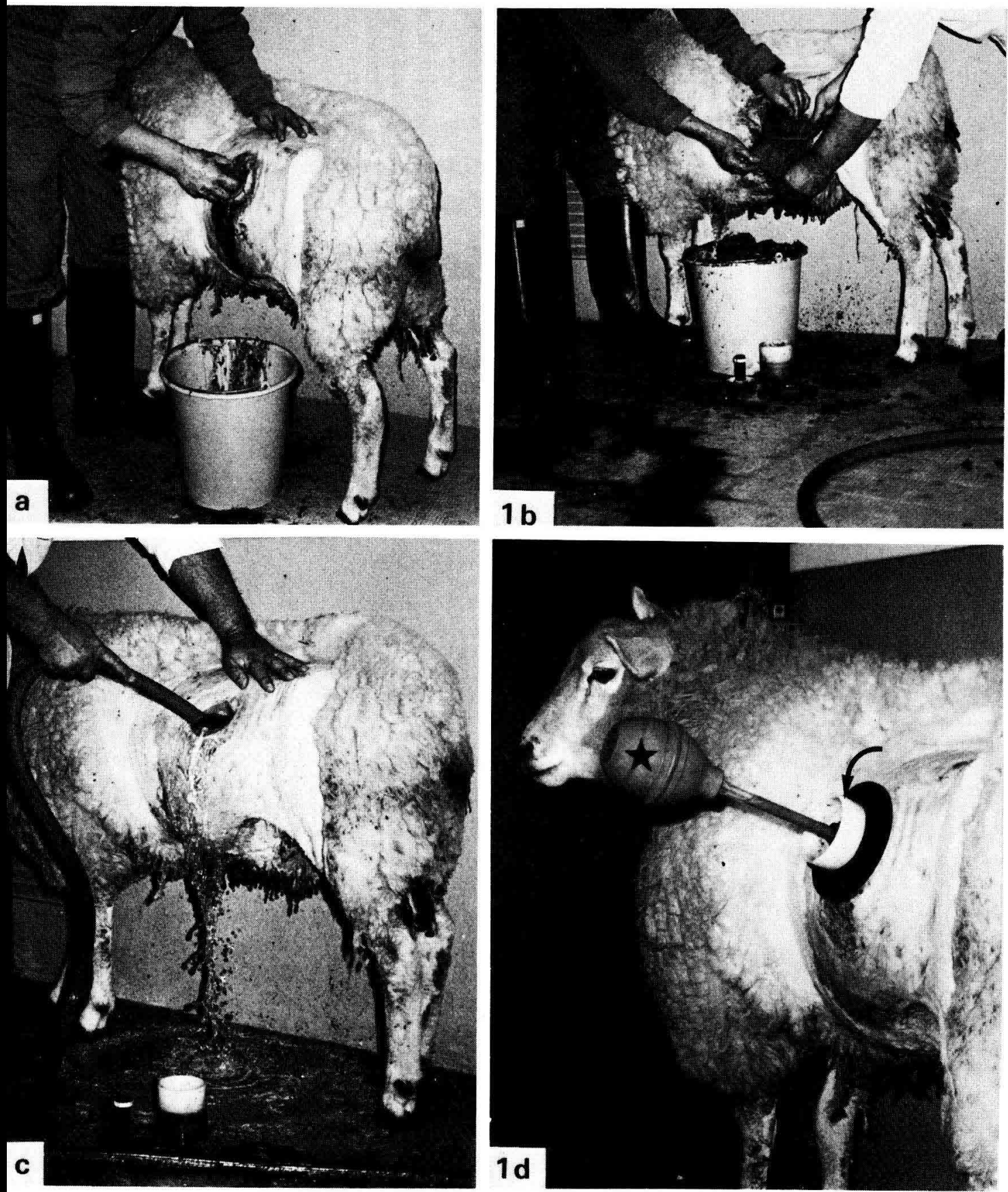
les premiers aliments ingérés. Elle est facilitée par la présence de Ruminants adultes placés à proximité. Cependant il est important d'éviter tout contact direct, ou même indirect, entre les animaux afin d'empêcher l'apparition de protozoaires ciliés dans les rumens défaunés. II est donc nécessaire d'observer une discipline stricte dans la manipulation de tels animaux (Williams ef Dinusson, 1972) aussi longtemps que l'on désire mainfenir les animaux à l'état défauné. L'absence de protozoaires ciliés est contrôlée régulièrement par l'observation au microscope de $0,05 \mathrm{ml}$ de contenu de rumen.

Résultats et justification de la méthode (tabl. 1).

La technique de défaunation est fiable puisque les 14 animaux traités ont été défaunés. Elle est également bien tolérée par les animaux qui ont pu être utilisés pour des études de digestion environ 3 semaines après le dernier vidage. Toutefois

TABLEAU 1

Comparaison des résultats obtenus entre les différentes techniques de défaunation étudiées

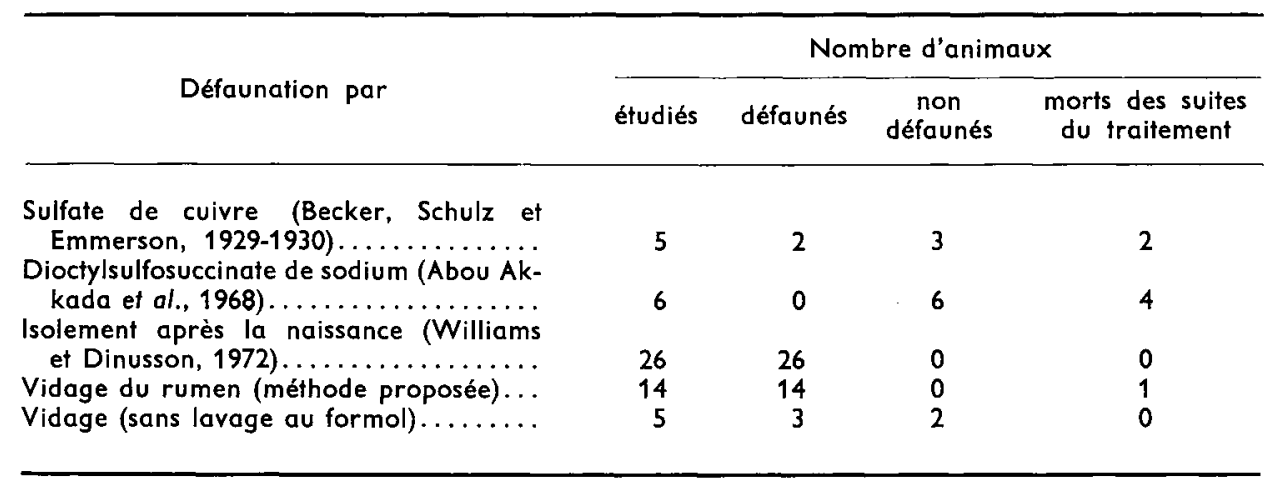

un mouton est mort. L'autopsie a révélé qu'une partie non négligeable de formol avait pénétré dans le feuillet, ce qui a provoqué une nécrose des lames et des lamelles, puis une infection au niveau de cet organe. Il est donc nécessaire de rincer avec soin la muqueuse du rumen après avoir laissé le formol peu de temps (environ $1 \mathrm{mn}$ ) en contact avec cette dernière. Dans le cas où l'on n'ajoute pas de formol à l'eau de lavage du rumen, le succès de l'opération devient aléatoire. Sur 5 animaux ainsi traités, 3 ont été totalement défaunés; chez les 2 autres moutons nous avons noté la présence d'une population d'Entodinium importante 2 semaines après le traitement.

En l'absence d'une thérapeutique appropriée, nous avons observé sur 10 animaux traités 2 cas de carence aiguë en vitamine $B$, et plus particulièrement en vitamine B1, à la fin de la période de jeûne ; c'est pourquoi nous recommandons l'administration de complexes vitaminiques au cours du traitement. De la même manière, nous avons constaté sur l'ensemble des moutons éłudiés une élévation de la valeur du $\mathrm{pH}$ du jus de rumen, dès qu'ils sont mis à jeun et que le rumen est vidé, au cours du 
processus de défaunation. $\mathrm{Ce} \mathrm{pH}$ élevé $(8,5)$, lié à la sécrétion salivaire et à l'absence de fermentations dans le rumen, peut être responsable de l'apparition de phénomènes de putréfactions indésirables et pouvant être toxiques pour les animaux. C'est pourquoi nous administrons régulièrement, au cours de la période de jeûne, une solution d'acide acétique dans le rumen.

La reprise de l'ingestion se fait, en général, spontanément dès la distribution d'aliment. Nous avons noté toutefois une sensibilité plus grande au jeûne prolongé chez les moutons de race Texel. Nous avons dû, chez ces derniers, introduire par l'intermédiaire de la fistule, un peu de fourrages grossiers sous forme longue dans le rumen ainsi que du propionate de sodium pour favoriser la reprise des contractions du rumen qui avaient disparues. L'implantation d'une population bactérienne rapidement abondante peut être favorisée par l'introduction d'un litre de contenu de rumen à $39^{\circ} \mathrm{C}$, provenant d'un autre mouton normalement fauné, qui a été préalablement congelé. Nous avons également remarqué que l'injection d'autolysats de poissons de mer pendant la durée du jeûne améliore l'état général des animaux et supprime en partie les difficultés liées à la reprise de l'ingestion.

\section{Conclusion.}

L'intérêt principal de la méthode que nous décrivons est qu'elle permet d'obtenir rapidement et d'une manière sûre des animaux défaunés. Nous n'avons toutefois pas de données précises sur la composition qualitative de la flore qui s'établit dans les rumens défaunés. L'absence de troubles digestifs ainsi que les performances zootechniques normales ef l'utilisation prolongée de tels animaux, permettent de penser que la population bactérienne est peu différente de celles des moutons conventionnels. Parmi l'ensemble des autres techniques étudiées, seule la méthode de l'isolation nous a permis d'aboutir à de bons résultats. Mais cette dernière méthode nécessite d'avoir une réserve importante d'animaux qui devront être élevés depuis la naissance jusqu'à l'âge adulte où ils pourront être utilisés pour des études de digestion. Ils devront donc être manipulés avec précaution pendant de longues périodes afin d'éviter toute contamination accidentelle. Comme le montrent nos résultats, les techniques de Becker, Schulz et Emmerson (1929-1930) et de Abou Akkada et al. (1968) sont peu fiables et dangereuses pour les animaux (tabl. 1).

Reçu en août 1978.

Accepté en décembre 1978.

\section{Références}

ABOU AKKADA A. R., BARTLEY E. E., BERUBE R., FINA L. R., MEYER R. M., HENRICKS D., JULIUS F., 1968. Simple method to remove completely ciliate protozoa of adult ruminants. Appl. Microbiol., 16, 1475-1477.

BECKER E. R., SCHULZ J. A., EMMERSON M. A., 1929-1930. Experiments on the physiological relationship between the stomach infusoria of ruminants and their hosts, with a bibliography. J. Sci., 4, 215-251.

CLARKE R. T. J., REID C. S. W., 1969. Bloat in cattle. XXXI. The effect of dimetridazole on the rumen ciliate protozoa of dry and lactating cows. N. Z. J. agric. Res., 12, 437-445. 
EADIE J. M., GILL J. C., 1971. The effect of the absence of rumen ciliate protozoa on growing lambs fed on a roughage-concentrate diet. Br. J. Nutr., 26, 155-167.

EADIE J. M., HOBSON P. N., MANN S. O., 1967. A note on some comparisons between the content of barley-fed steers and that of young calves also fed on a high concentrate ration. Anim. Prod., 9, 247-250.

EADIE J. M., HYLDGAARD-JENSEN J., MANN S. O., REID R. S., WHITELAW F. G., 1970. Observations on the microbiology and biochemistry of the rumen in cattle given different quantities of a pelleted barley ration. Br. J. Nutr., 24, 157-177.

EADIE J. M., OXFORD A. E., 1954. A remarkable disintegrative effect of skatole upon certain rumen ciliale protozoa. Nature, 174, 973.

HUNGATE R. E., 1966. The rumen and its microbes. Acad. Press N.-Y., San Francisco, London.

KURIHARA Y., EADIE J. M., HOBSON P. N., MANN S. O., 1968. Relationship between bacteria and ciliate protozoa in the sheep rumen. J. gen. Microbiol., 51, 267-288.

SENAUD J., JOUANY J. P., GRAIN J., DE PUYTORAC P., 1973. Dynamique d'une population de Polyplastron multivesiculum (cilié «oligotriche") en équilibre dans le rumen du mouton. C. R. Acad. Sci. Paris, Sér. D, 277, 197-200.

WILLIAMS P. P., DINUSSON W. E., 1972. Composition of the ruminal flora and establishment of ruminal ciliated protozoal species in isolated calves. J. anim. Sci., 34, 469-474.

WILLIAMS P. P., DINUSSON W. E., 1973. Ruminal volatile fatty acid concentrations and weight gains of calves reared with and without ruminal ciliated protozoa. J. Anim. Sci., 36, 588-591.

WRIGHT D. E., CURTIS M. W., 1976. Bloat in cattle XLII. - The action of surface-active chemicals on ciliated protozoa. N.Z.J. agric. Res., 19, 19-23.

ZAINAB B., 1978. Contribution à l'étude des interrelations entre trois genres de profozoaires ciliés du rumen de moufon : dynamique des populations; rôle dans l'utilisation d'un aliment aggloméré (luzerne/orge : 40/50). Thèse $3^{e}$ cycle, $n^{\circ} 545,68$ pp., Univ. Clermont II. 